\title{
The Long-term Variation in the RV Tauri Star U Mon
}

\author{
Karen R. Pollard ${ }^{1}$ and P. L. Cottrell \\ Mt John University Observatory, Department of Physics \& Astronomy, \\ University of Canterbury, Christchurch, New Zealand
}

\section{Introduction}

The RV Tauri stars are semiregular pulsating variables located in the brightest part of the Cepheid II instability strip. They have a characteristic light curve of alternating deep and shallow minima. A subset of the RV Tauri stars (the RVb subclass) exhibit long-term (500 to 2600 day) light and radial velocity variations. Although it is well established that the short-term variations are due to pulsations, the long-term behaviour is not well understood.

\section{Observations and results}

$B V R I$ photometry and high-resolution spectra of $\mathrm{U}$ Mon (the brightest member of the RVb subclass) were obtained at the Mt John University Observatory (MJUO) between 1990 Aug and 1994 May. The light and colour curves obtained clearly show the long-term variation in U Mon (Fig. 1(a) and (b)). The reddest colours occur slightly later than the long-term minimum in the light curve. The short-term light and colour variations are 'damped' at the long-term minimum.

The MJUO radial velocities were analysed together with those previously published for U Mon in order to obtain an orbital solution. The derived orbital period of $2597 \mathrm{~d}$ is in reasonable agreement with the visual light period of $2475 \mathrm{~d}$ (Percy 1993). The orbit is eccentric $(e=0.43)$ with periastron at HJD 2448349.

Strong $\mathrm{H} \alpha$ emission is seen around pulsational phases 0.15 and 0.65 , corresponding to when the propagation of shock waves through RV Tauri star photospheres is observed to occur (Lèbre \& Gillet 1991). Additional H $\alpha$ emission is seen during HJD 2448400 to 2448600 (Fig. 1(c)), when U Mon is faint, reddened and is closest to the earth in its orbit.

\section{Discussion}

Fokin (1994) suggests that the long-term variation in the RVb stars may be due to previously-ejected dust eclipsing the star at certain orbital phases. Using a model proposed for the HR4049 system by Waelkens \& Waters (1993), Percy (1993) suggests that the long-term variation in U Mon may be due to the star being eclipsed by the inner edge of a dust torus which surrounds the binary system. The light decrease and the reddening of the colours in U Mon

\footnotetext{
${ }^{1}$ Present address: South African Astronomical Observatory, PO Box 9, Observatory 7935, Cape.
} 


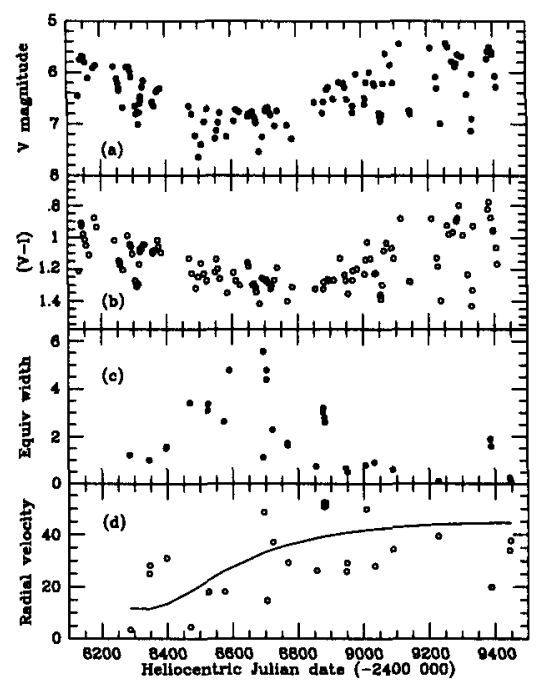

Figure 1. MJUO observations of U Mon: (a) the $V$ light curve; (b) $(V-I)$ colour curve; (c) $\mathrm{H} \alpha$ equivalent width; (d) radial velocity curve, showing MJUO radial velocities (o) and the orbital solution (-).

appears consistent with both models. One effect that is difficult to explain by either model is the 'damping' of the short-term light and colour variations at long-term minimum.

An alternative explanation for the long-term variation is that the binary system involving U Mon is interacting at certain orbital phases. The enhanced $\mathrm{H} \alpha$ emission is attributed to mass loss or transfer which occurs shortly after periastron due to the effect of the binary interaction. The pulsational variations will be damped at this orbital phase. U Mon appears reddened due to the presence of gas and dust from a previous or current mass loss event, the binary interaction or possibly tidal forces. It is quite possible in a system where circumstellar is known to exist, that a combination of obscuration and interaction mechanisms is present.

Acknowledgments. The authors wish to thank P. Kilmartin \& A. Gilmore for obtaining the photometry and $\mathrm{M}$. Albrow for obtaining a number of spectra.

\section{References}

Fokin, A.B. 1994, A\&A, 292, 133

Lèbre, A., \& Gillet, D. 1991, A\&A, 246, 490

Percy, J.R. 1993, in: Luminous High-Latitude Stars, D.D. Sasselov, ASP Conf. Series 45, 295

Waelkens, C., \& Waters, L.B.F.M. 1993, in Luminous High-Latitude Stars, D.D. Sasselov (ed.), ASP Conf. Series 45, 219 